\title{
Psychometric Properties of the Sleep Questionnaire
}

\section{Davood Manavipour*}

Department of Psychology, Garmsar Branch, Islamic Azad University, Garmsar, Iran

\section{A BSTRACT}

Introduction: Sleep is an important part of human life. Sleep disorders are very common and have direct and indirect impacts on other aspects of life. Detection of sleep disorders is very important and needs the appropriate tools. This study was aimed to determine the psychometric properties of questionnaire on sleep disorders. Materials and Methods: Using correlation statistical method, the sleep condition of 500 students from Garmsar University was evaluated by psychometric properties of the sleep questionnaire. Results: The reliability test using Cronbach's alpha was 0.79 . Exploratory factor analysis for Validity revealed that $61 \%$ of the variance in scores of questions about sleep disorders could be explained by the scale. After varimax rotation, two major forms of sleep disorders, insomnia and hypersomnia, were identified. Confirmatory factor analysis of indicators of structural equation model for validity showed that the model is fitted to the data. Conclusion: This questionnaire can be used for measurement and screening of sleep disorders for both research and treatment proposes.

* Corresponding Author: Davood Manavipour

E-mail: Manavipor53@yahoo.com

\section{Key words:}

1. Psychometrics

2. Sleep

3. Sleep Initiation and Maintenance Disorders

4. Disorders of Excessive Somnolence 


\section{ويثَى هاى روانسنجى يرسشنامؤ اختلال خواب}

داود معنوى يور"

گروه روانشناسى، واحد كرمسار، دانشعاه آزاد اسلامى، گرمسار، ايران

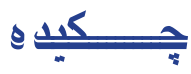

مقدمه: خواب يك بخش مهمى از زندگى انسان است. اختلالات خواب بسيار شايع هستند و اثرات اترات

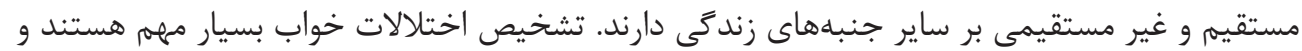

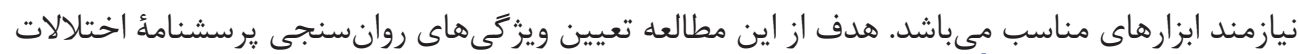

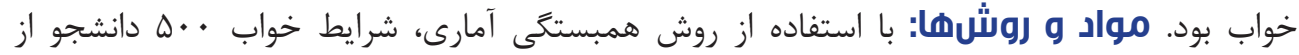

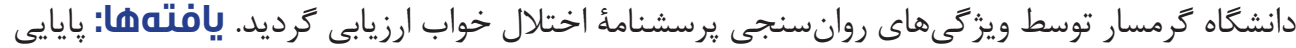

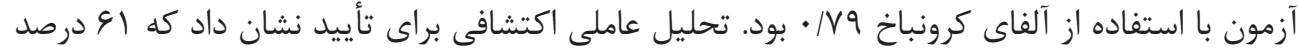

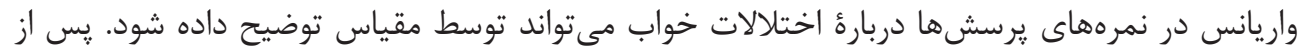

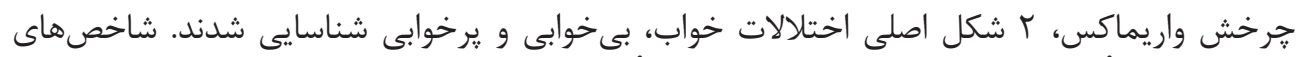

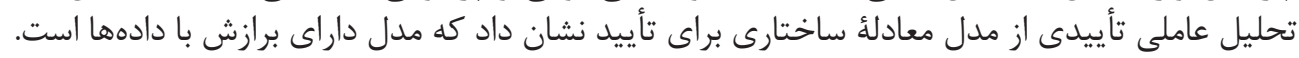

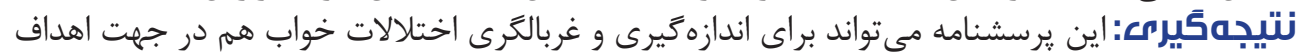

$$
\text { تحقيقى و هم درمانى مورد استفاده قرار گيردان }
$$

ب. ب. اختلالات شروع و دوام خواب

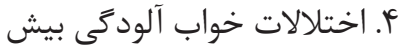




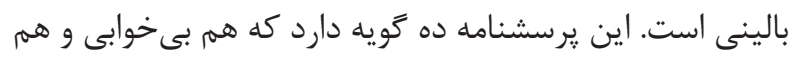

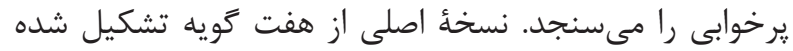

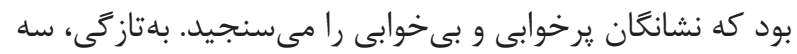

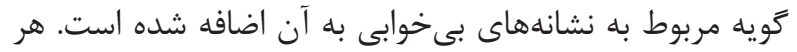

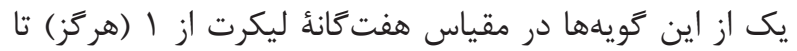

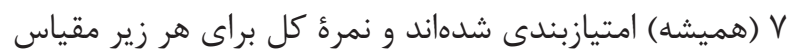

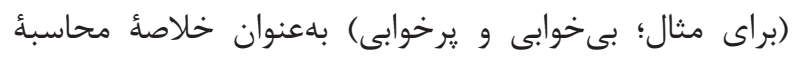

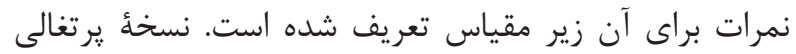

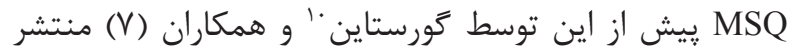

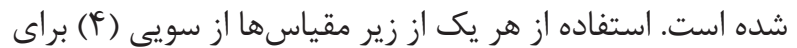

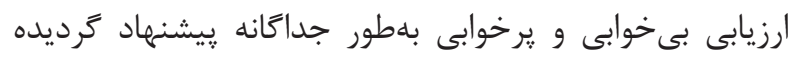

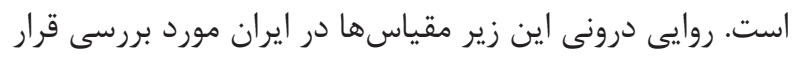

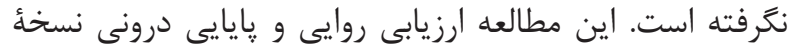

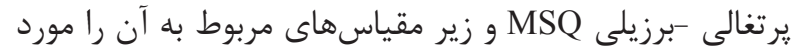

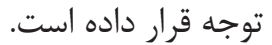

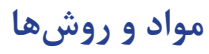

يزوهش حاضر در قالب يزوهشى توصيفى -همبستكى انجام

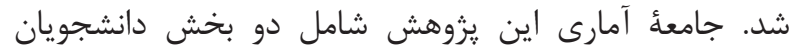

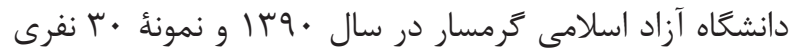

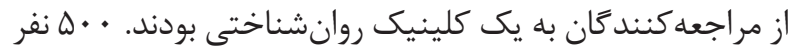
از دانشجويان به شيؤ در دستر بـ كرين انتخاب شدند.

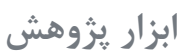

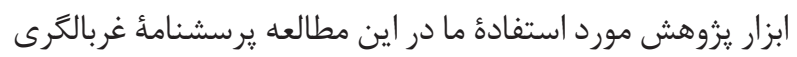

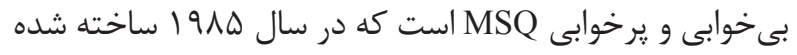

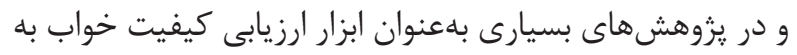

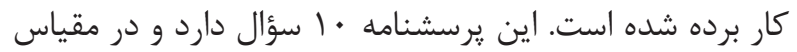

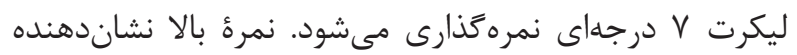

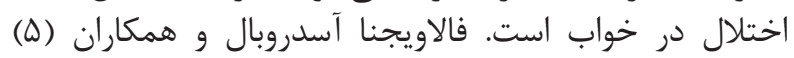

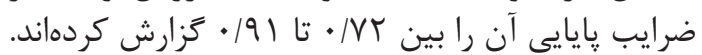

بافتهها

براى برآورد پايايى يرسشنامه از روش آلفاى كرونباخ استفاده

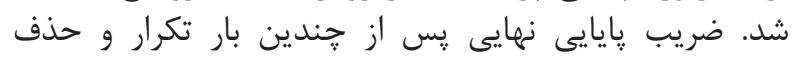

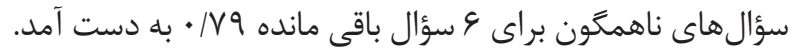

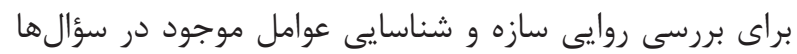

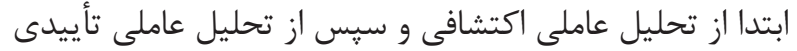

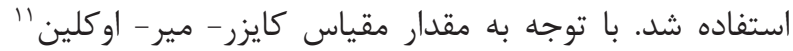

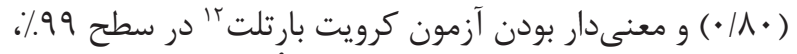

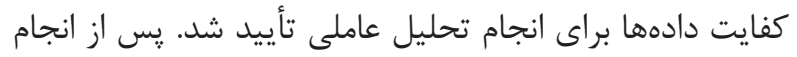

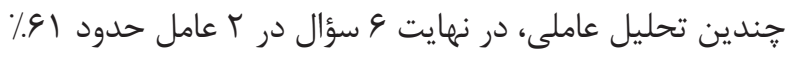

از واريانس اختلالات خواب را تبيين كردند (نمودار ()).

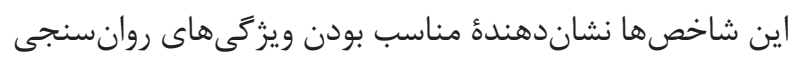

${ }^{1}$ Combs and Krippner

${ }^{2}$ Repair-restoration theory

${ }^{3}$ Evolutionary-circadian theory

${ }^{4}$ Wish-fulfillment

${ }^{5}$ Activation-synthesis hypothesis

${ }^{6}$ Asdrubal Falavigna

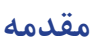

بى خوابى از مسايلى است كه باعث بروز اختلال در زندكى إنى و

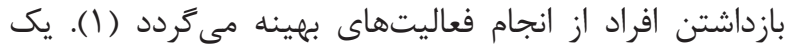

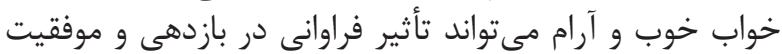

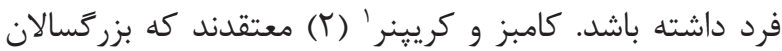

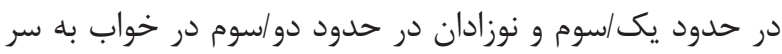

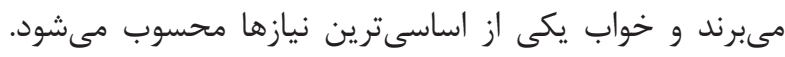

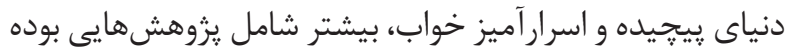

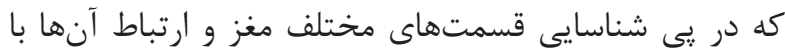

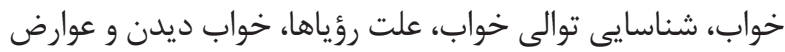

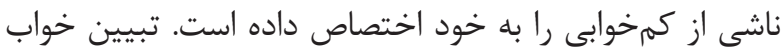

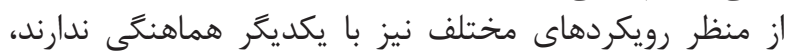
بهوَّهاى كه نظريههاى متفاوتى دربارئ آن ارائه شده است.

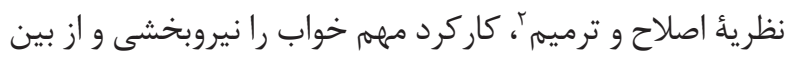

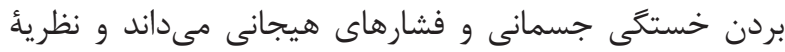

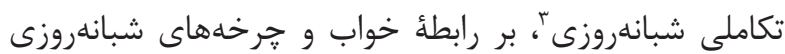

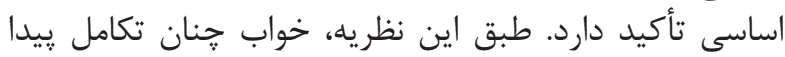

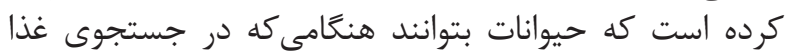

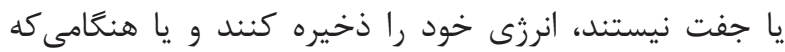

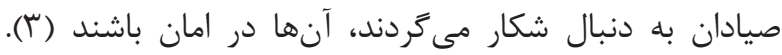

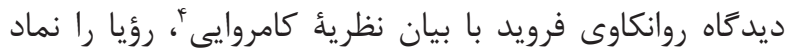

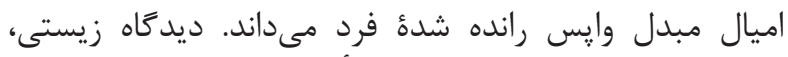

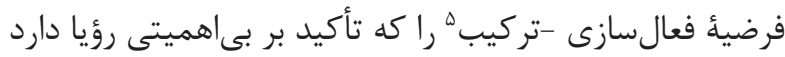

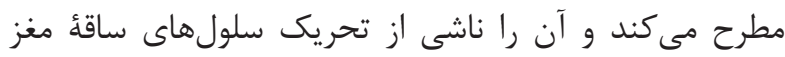

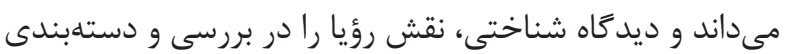

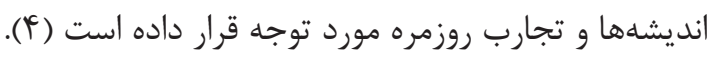

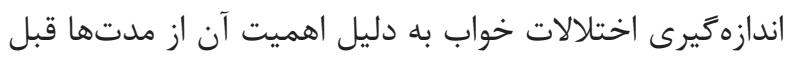

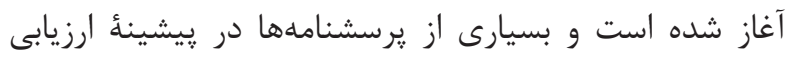

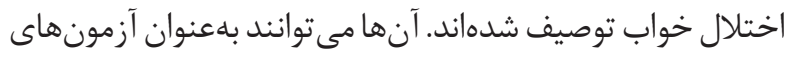

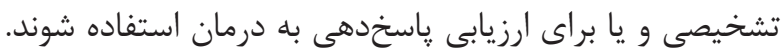

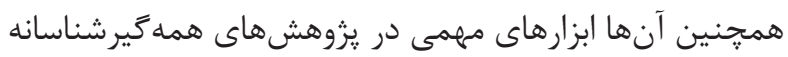

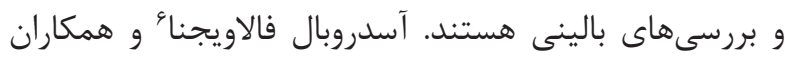

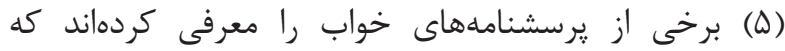

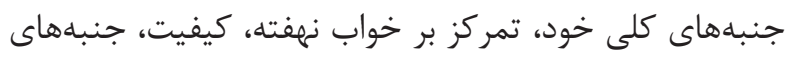

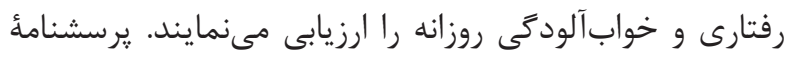

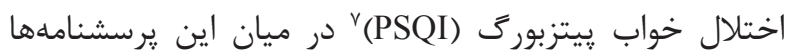

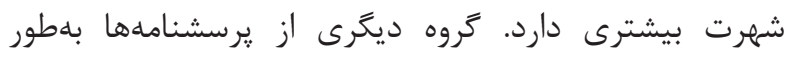

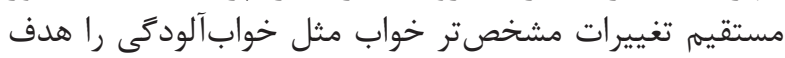

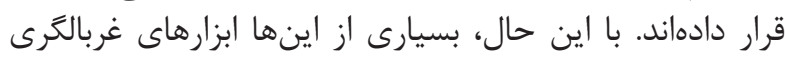

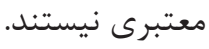

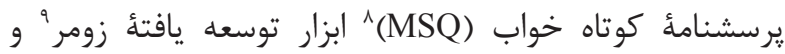
همكاران (9) براى غربالكَرى اختلالات خواب در جمعيتهائ ترائ

\footnotetext{
${ }^{7}$ Pittsburgh sleep quality index

${ }^{8}$ Mini sleep questionnaire

${ }^{9}$ Zomer

${ }^{10}$ Gorestein

${ }^{11}$ Kaiser-Meyer-Oklin measure (KMO)

${ }^{12}$ Bartlett's test of sphericity
} 


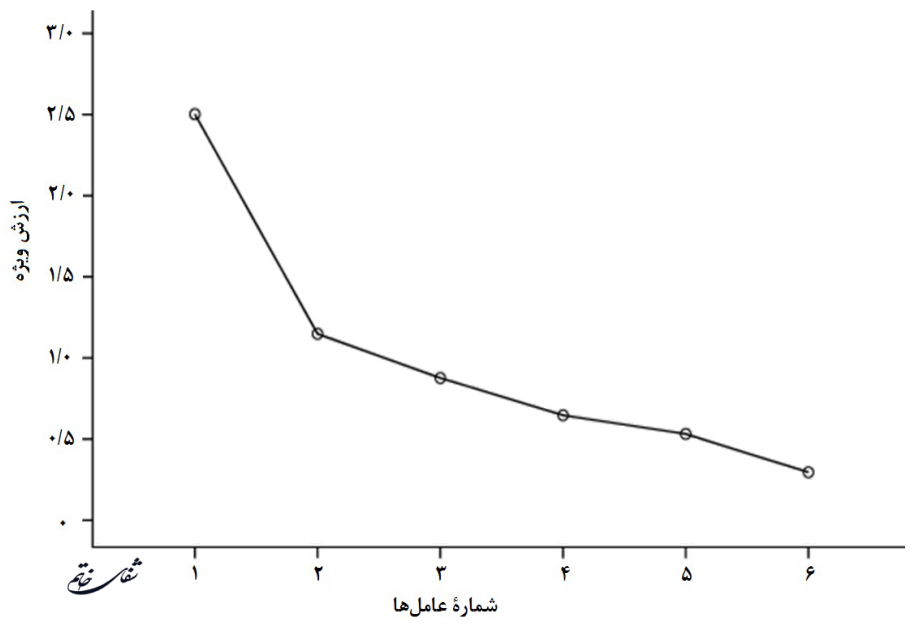

نمودار ا- آزمون اسكرى كتل براى تعيين عوامل.

شاخص برازش خى دو به درجأ آزادى نشان مى دهدد كه مدل

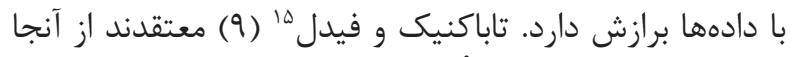

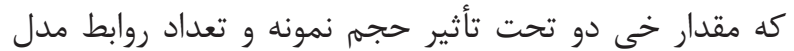

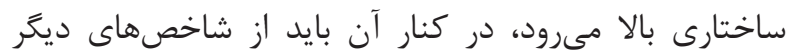

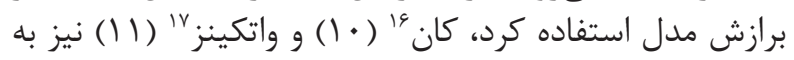

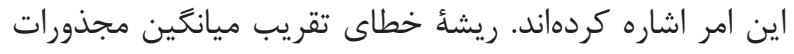
(RMSEA)

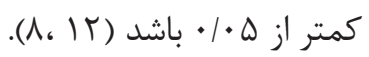

آزمون است. از ماتريس همبستكى بين برسش ها و عامل ها پس

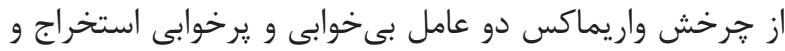
نام كذارى شدند.

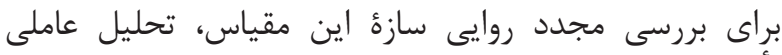

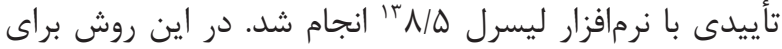

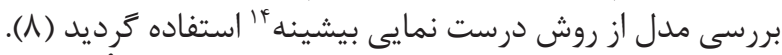

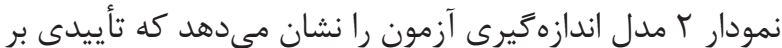
نتايج ييشين است

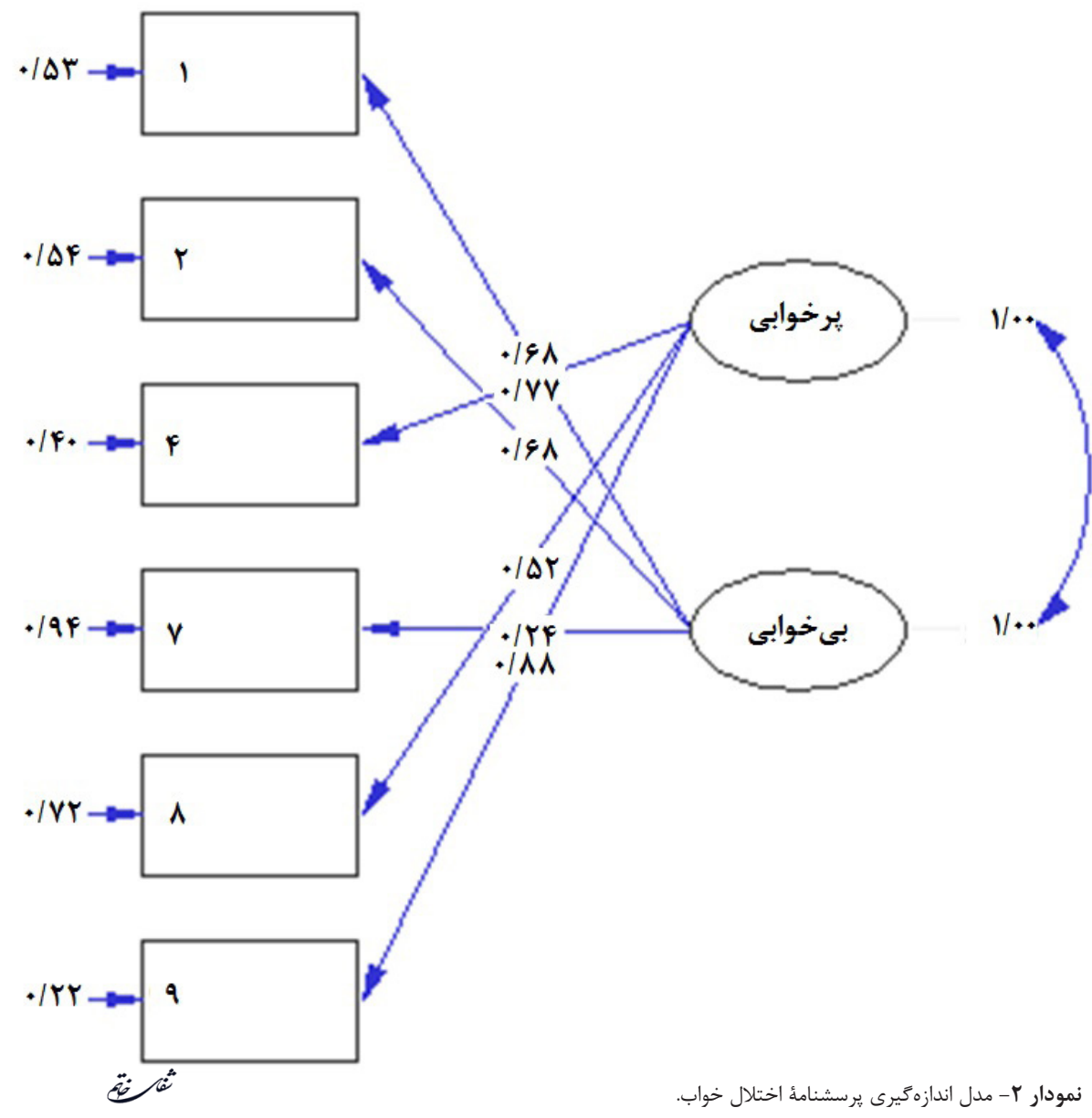




\begin{tabular}{|c|c|c|c|c|c|c|c|c|c|}
\hline RMSEA & P-Value & df & Chi-Square & CFI & GFI & AFGI & NFI & NNFI & \\
\hline qqu & $\cdot / \cdot V$ & $\Lambda$ & $\mid r / \cdot 1$ &.$/ 99$ &.$/ 99$ &.$/ 9 V$ &.$/ 91$ &.$/ 91$ & ز ش \\
\hline
\end{tabular}

\begin{tabular}{|c|c|c|c|c|c|c|c|}
\hline ضريب & بار عاملى اكتشافى & مجنور & ضريب مسير & عامل & سؤالها & قديم سؤال & جديد سؤال \\
\hline$\cdot 149$ & $\cdot / V_{\Delta}$ & $\cdot 144$ & $\cdot 191$ & \multirow{3}{*}{ 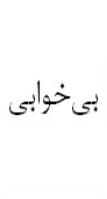 } & مشكل در به خواب رفتن & 1 & 1 \\
\hline$\cdot 149$ & $\cdot / V T$ & $\cdot 149$ & $\cdot 191$ & & مكرراً از خواب يريدن & r & r \\
\hline$\cdot / 49$ & $\cdot 194$ & $.1 \cdot 9$ & $\cdot / \pi t$ & & خيلى زود بيدار شدن از خواب & V & r \\
\hline.$|9|$ & $\cdot / \Lambda F$ & $\cdot 109$ & $\cdot / V V$ & \multirow{3}{*}{ 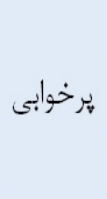 } & خوابآلودكَى بيش از حد در روز & k & r \\
\hline$\cdot|Q|^{c}$ & $\cdot / V T$ & $\cdot / 4 V$ & $\cdot / \Delta r$ & & سردرد در هنغام بيدارى & $\Lambda$ & $\Delta$ \\
\hline $\begin{array}{l}\cdot \mid q k \\
0\end{array}$ & $\cdot / 1 \Delta$ & $\cdot / \vee \wedge$ & $\cdot / 1 \Lambda$ & & خوابآلودكى در طول روز & 9 & 9 \\
\hline
\end{tabular}

خواب است و شاخصهاى روانسنجى آن در مطالعات متعدد

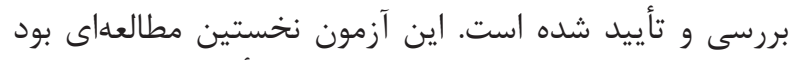

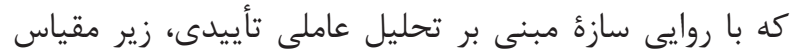

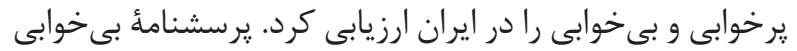

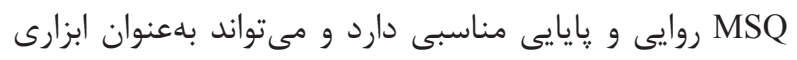

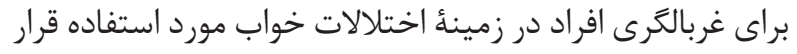

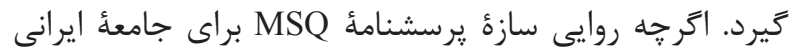

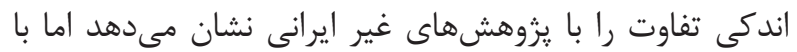

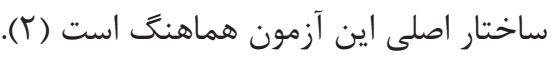

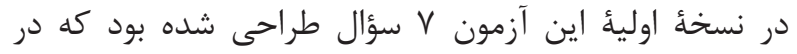

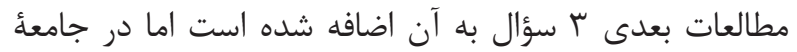

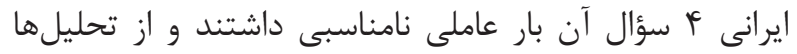

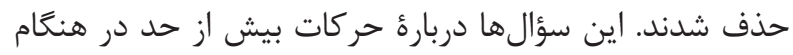

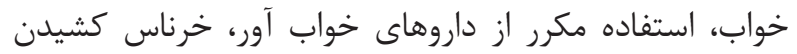

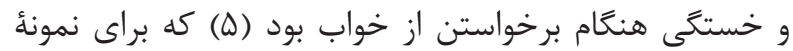

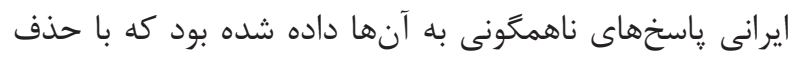

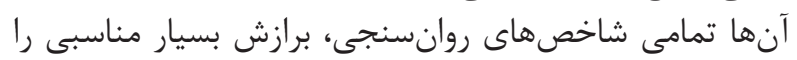

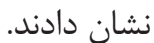

$$
\text { تشكر و قدردانى }
$$

از دانشجويان كوشا خانمها؛ سمانه ايجى، فاطمه عباسى و و محدثه يازوكى كه در زردآورى و ورود متقبل شدهاند، تشكر مى كنه.

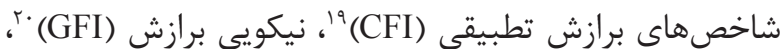

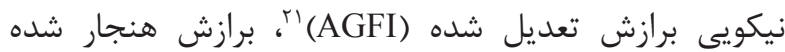
(NFI)

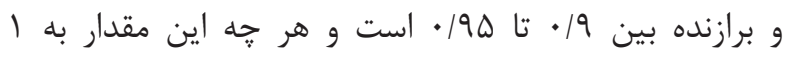

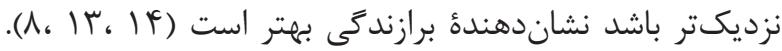

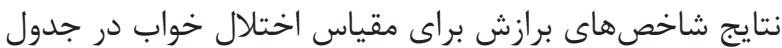

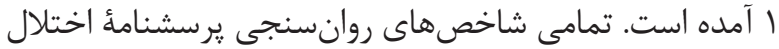
خواب در جداول ا و ب آمده است. تمامى شاخصها اعم از آلفاى كرونباخ، تحليل عاملى اكتشافى

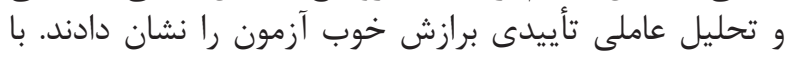

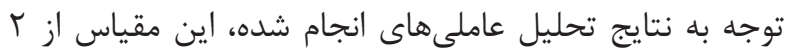

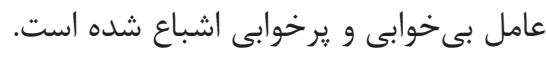

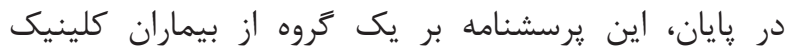

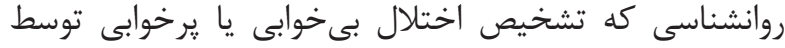

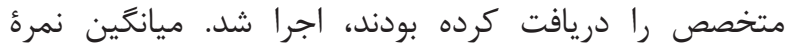

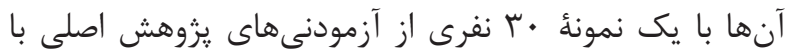

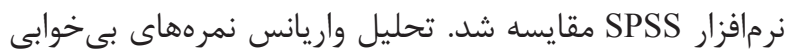

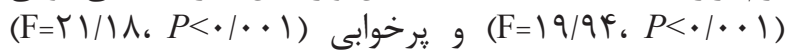

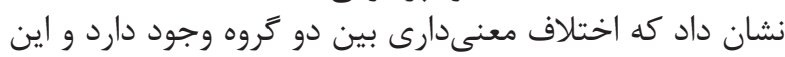

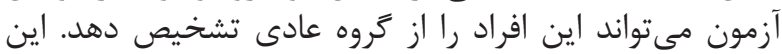
يافته نيز تأييدى بر روايى افتراقى يرسشن إنى رامه است.

$$
\text { بحث و نتيجه كيرى }
$$

يرسشنامةُ برزيلى MSQ ابزار مناسبى براى غربالكرى اختلالات

\footnotetext{
${ }^{19}$ Comparative fit index

${ }^{20}$ Goodness of fit index

${ }^{21}$ Adjust goodness of fit index
}

${ }^{22}$ Normed fit index

${ }^{23}$ Non-normed fit index 
1. Buysse DJ, Angst J, Gamma A, Ajdacic V, Eich D, Rössler W. Prevalence, course, and comorbidity of insomnia and depression in young adults. Sleep. 2008; 31(4): 473-80.

2. Combs A, Krippner S. Dream sleep and waking reality: A dynamical view of two states of consciousness. In: Toward a Science of Consciousness 1996. MIT Press, Cambridge. 1996.

3. Hobson JA. The dreaming brain. Basic Books. 1989.

4. Shochat T, Tzischinsky O, Oksenberg A, Peled R. Validation of the pittsburgh sleep quality index hebrew translation (PSQI-H) in a sleep clinic sample. Isr Med Assoc J. 2007; 9(12): 853-6.

5. Falavigna A, de Souza Bezerra ML, Teles AR, Kleber FD, Velho MC, da Silva RC, et al. Consistency and reliability of the brazilian portuguese version of the mini-sleep questionnaire in undergraduate students. Sleep Breath. 2011; 15(3): 351-5.

6. Zomer J, Peled R, Rubin AH, Lavie P. Mini-sleep questionnaire (MSQ) for screening large populations for EDS complaints. Gustav Fischer Verlag New York. 1985; p. 467-70.
7. Gorestein C, Tavares S, Aloe F. Sleep self-evaluation questionnaires. Clinicalevaluationscalesinpsychiatryand psycopharmacology. Lemos, Sao Paulo. 2000; p. 423-34.

8. Hooman HA. Structural equation modeling with Lisrel application. Tehran: Samt. 2005.

9. Tabachnick BG, Fidell LS. Using multivariate statistics. 5th ed. New York, Allyn and Bacon. 2007.

10. Kahn JH. Factor analysis in counseling psychology research, training, and practice: principle, advances, and application. Couns Psychol. 2006; 34(5): 684-718.

11. Watkins MW. Mac parallel analysis (computer software). State College, PA. 2000.

12. Brown MW, Cudeck R. Alternative ways of assessing model fit. Sociol Methods Res. 1992; 21(2): 230-58.

13. Henson RK, Roberts JK. Use of exploratory factor analysis in published research: common errors and some comment on improved practice. Educ Psychol Meas. 2006; 66(3): 393-416.

14. Kline RB. Principle and practice of structural equation modeling. New York: Guilford press. 2005. 